\title{
Loop-mediated isothermal amplification (LAMP) assay for the diagnosis of fasciolosis in sheep and its application under field conditions
}

\author{
María Martínez-Valladares ${ }^{1,2^{*}}$ and Francisco Antonio Rojo-Vázquez ${ }^{1,2}$
}

\begin{abstract}
Background: Loop-mediated isothermal amplification (LAMP) is a very specific, efficient, and rapid gene amplification procedure in which the reaction can run at a constant temperature. In the current study we have developed a LAMP assay to improve the diagnosis of Fasciola spp. in the faeces of sheep.

Findings: After the optimisation of the LAMP assay we have shown similar results between this technique and the standard PCR using the outer primers of the LAMP reaction. In both cases the limit of detection was 10 pg; also, the diagnosis of fasciolosis was confirmed during the first week post-infection in experimental infected sheep by both techniques. In eight naturally infected sheep, the infection with $F$. hepatica was confirmed in all animals before a treatment with triclabendazole and on day 30 post treatment in two sheep using the LAMP assay; however, when we carried out the standard PCR with the outer primers, the results before treatment were the same but on day 30 post-treatment the infection was only confirmed in one out of the two sheep. On the other hand, the standard PCR took around $3 \mathrm{~h}$ to obtain a result, comparing with $1 \mathrm{~h}$ and $10 \mathrm{~min}$ for the LAMP assay.
\end{abstract}

Conclusions: The LAMP assay described here could be a good alternative to conventional diagnostic methods to detect $F$. hepatica in faeces since it solves the drawbacks of the standard PCR.

Keywords: Fasciola spp, Sheep, Diagnosis, PCR, Loop-mediated isothermal amplification (LAMP)

\section{Background}

Species of Fasciola cause a zoonotic disease that is important from both economic and medical points of view in animals, principally ruminants. Its prevalence is increasing due to climate change, anthelmintic resistance [1] and man-made environmental modifications [2].

Diagnosis of fasciolosis is confirmed by the observation of parasite eggs in the faeces $[3,4]$ from seven weeks post-infection (wpi) onwards [5, 6]. Molecular methods were developed to increase the sensitivity and the specificity of conventional diagnosis, including immunological techniques [6]. However, PCR is only available to particular facilities because of the need for

\footnotetext{
*Correspondence: mmarva@unileon.es

${ }^{1}$ Faculty of Veterinary Medicine, University of León, Campus de Vegazana, 24071 León, Spain

${ }^{2}$ Instituto de Ganadería de Montaña (CSIC-ULE), Finca de Marzanas, 24346 Grulleros, León, Spain
}

special detection devices. Another molecular technique called loop-mediated isothermal amplification (LAMP) is an alternative to PCR. LAMP assay is a very specific, efficient and rapid gene amplification procedure in which the reaction can run at a constant temperature [7]. In the current study we detected fasciolosis in experimentally and naturally infected sheep using the LAMP assay.

\section{Methods}

Seven sheep were experimentally infected with 200 metacercariae of Fasciola hepatica each. Faecal samples were collected from rectum weekly from the day of infection until 8 wpi. Ethical approval was obtained from Insituto de Ganadería de Montaña ethics committee complying with national regulations (R.D. 53/2013). We also analysed by molecular methods the individual level 
of infection of 8 naturally infected sheep, previously tested by Robles-Pérez et al. [8], before and after 30 days post-treatment $(\mathrm{dpt})$ with triclabendazole. The number of $F$. hepatica eggs was calculated by a sedimentation method.

At least $1 \mathrm{~g}$ faeces per sheep was collected weekly for a pool of faeces of all experimentally infected animals. DNA extraction was applied to $0.5 \mathrm{~g}$ of each weekly pool using "SpeedTools Tissue DNA Extraction Kit" (Biotools, Madrid, Spain. Regarding natural infection, DNA samples from faeces collected in the study by RoblesPérez et al. [8] were analysed.

DNA from adult worms was also extracted as previously is indicated in faeces. Serial 10-fold dilutions from F. hepatica DNA were prepared ranging from $10^{-1} \mathrm{ng} / \mu \mathrm{l}$ to $10^{-6}$ $\mathrm{ng} / \mu \mathrm{l}$ to determine the sensitivity. A sample with $10^{-1} \mathrm{ng} /$ $\mu \mathrm{l}$ DNA was used as a positive control in all reactions. DNA from other helminths (Dicrocoelium dendriticum, Calicophoron daubneyi, Teladorsagia circumcincta, Haemonchus contortus and Trichostrongylus colubriformis) was also extracted to determine the specificity.

Primers used for the LAMP assay were designed based on a highly conserved region of Fasciola spp. genome. Genbank sequences (http://blast.ncbi.nlm.nih.gov/ Blast.cgi) (Table 1) including the internal transcribed spacer 2 (ITS2) region were tested in silico through BLAST searches and alignment analysis using the ClustalW module of the MegAlign software (DNASTAR Inc., Madison, WI, USA). A 568 base pairs (bp) consensus sequence was selected for the design of specific primers using Primer Explorer V4 (http://primerexplorer.jp/e/). Two sets of primers comprising two outer (F3 and B3) and two inner (FIP and BIP) were selected. FIP contained the F1c (complementary to F1) and F2 sequences.

Table 1 GenBank accession numbers for the different Fasciola spp. isolates included in the alignment

\begin{tabular}{ll}
\hline Isolates & GenBank accession number \\
\hline Fasciola sp. & JF708043 \\
F. hepatica & GQ231547 \\
F. hepatica & JF708026 \\
F. hepatica & JF708036 \\
F. hepatica & $\mathrm{HM746786}$ \\
F. hepatica & $\mathrm{AM} 709622$ \\
F. hepatica & $\mathrm{JF} 432071$ \\
F. gigantica & $\mathrm{JF} 432074$ \\
F. gigantica & $\mathrm{JF} 496714$ \\
F. gigantica & $\mathrm{KF} 425321$ \\
F. gigantica & $\mathrm{AM} 850108$ \\
F. gigantica & $\mathrm{HM} 746788$ \\
F. gigantica & $\mathrm{JN} 828956$ \\
\hline
\end{tabular}

BIP contained the B1c (complementary to B1) and B2 sequences (Fig. 1 and Table 2).

A standard PCR, using $0.5 \mu \mathrm{M}$ of primers F3 and B3 (Table 2) and $1 \mu \mathrm{l}(20 \mathrm{ng} / \mu \mathrm{l})$ DNA, was carried out in a total volume of $20 \mu \mathrm{l}$ following previously published protocol [6] with annealing temperature of $63{ }^{\circ} \mathrm{C}$ for $30 \mathrm{~s}$. Amplification products were analysed by electrophoresis in $2 \%$ agarose TBE (Tris base, boric acid and EDTA). All reactions were carried out in triplicate. One of the resulting bands was sequenced at the Laboratorio de Técnicas Instrumentales (León, Spain).

LAMP assay was set up testing different betaine, $\mathrm{MgSO}_{4}$ concentrations and temperatures. LAMP reaction mixtures $(25 \mu \mathrm{l})$ contained $40 \mathrm{pmol}$ of each FIP and BIP primers, 5 pmol of each F3 and B3 primers, $1.4 \mathrm{mM}$ of each dNTPs (Biotools, Spain), 1× Isothermal Amplification Buffer (New England Biolabs, Beverly, MA, USA), betaine $(0.8,1,1.2,1.4$, or $1.6 \mathrm{M})$ (Sigma, city, USA), $\mathrm{MgSO}_{4}(4,6,8$, or $10 \mathrm{mM})$ (New England Biolabs, UK) and $8 \mathrm{U}$ of Bst 2.0 WarmStart DNA polymerase (New England Biolabs, UK), with $1 \mu \mathrm{l}$ of DNA (20 ng/ $\mu \mathrm{l})$. Different temperatures were tested using a thermocycler (Bio-Rad) set at 61,63 , and $65^{\circ} \mathrm{C}$ for $60 \mathrm{~min}$ and then at $80{ }^{\circ} \mathrm{C}$ for $10 \mathrm{~min}$. Amplifications were visually detected by adding $2 \mu \mathrm{l}$ of $1: 10$ diluted $10,000 \times$ concentration fluorescent dye SYBR Green I (Invitrogen, Carlsbad, CA USA). Green fluorescence was observed in successful LAMP reactions; it remained orange in the negative reactions. The LAMP reaction products were also analysed by electrophoresis in $2 \%$ agarose TBE. All reactions were done in triplicate.

\section{Results}

In silico comparisons of LAMP primers showed a homology of $100 \%$ with F. hepatica and F. gigantica. The specificity of the outer primers, F3 and B3, was carried out by means of a standard PCR and LAMP reaction (Fig. 2). Primers amplified a band of 220 bp; after sequencing we showed a $100 \%$ homology with the sequence shown in Fig. 1, consensus sequence of the ITS2 region of Fasciola spp . The optimisation of the LAMP reaction was done with $1 \mathrm{M}$ betaine, $8 \mathrm{mM} \mathrm{MgSO}_{4}$ and incubation at $63{ }^{\circ} \mathrm{C}$.

The limit of detection of LAMP reaction was $10^{-3} \mathrm{ng} /$ $\mu \mathrm{l}$ (Fig. 3a); the amplification products were visualised on an agarose gel as a ladder of multiple bands (Fig. 3b). The sensitivity of the standard PCR with the primers F3 and $\mathrm{B} 3$ was also $10^{-3} \mathrm{ng} / \mu \mathrm{l}$ (Fig. $3 \mathrm{c}$ ).

In relation to the experimental infection, the first egg excretion was detected at 8 wpi and only in two sheep with a mean of 30 eggs per gram (epg); at 10 wpi the infection was confirmed in all animals. Using the LAMP assay and the standard PCR, the infection was detected from the first wpi onwards (Fig. 4a, b respectively). 


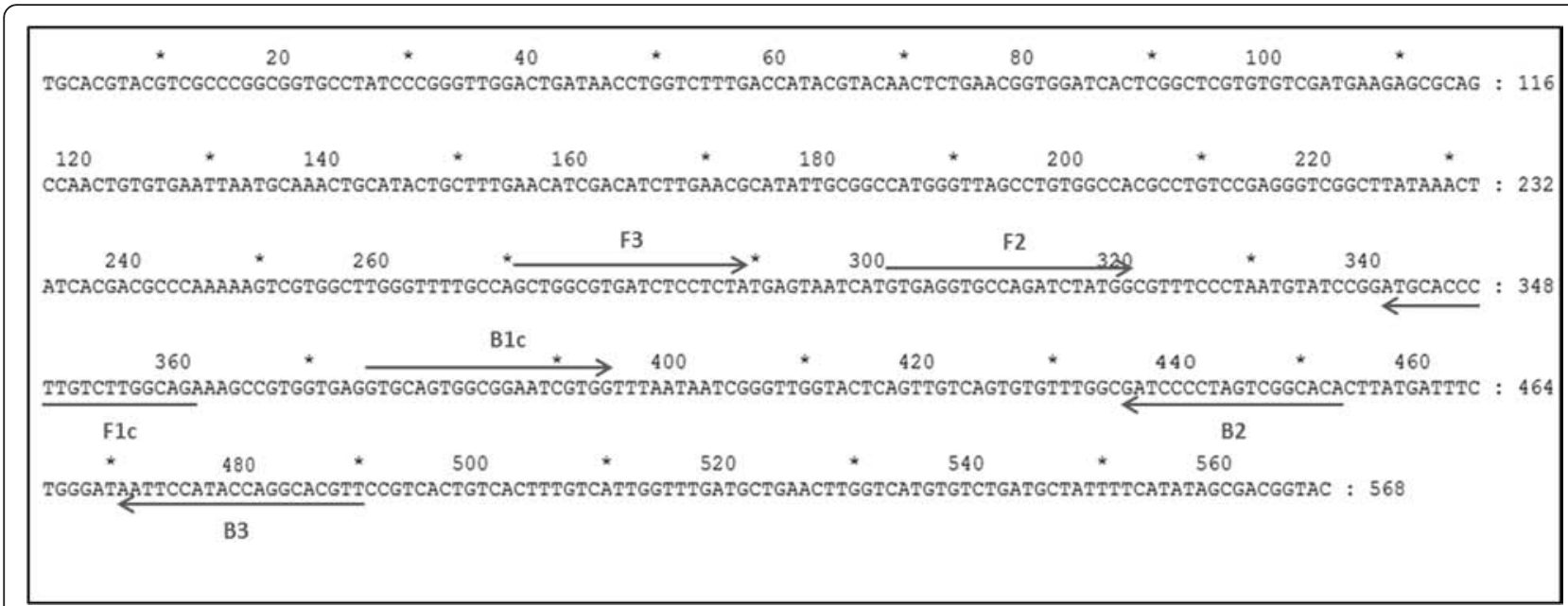

Fig. 1 LAMP primer set targeting the consensus sequence of the internal transcribed spacer 2 (ITS2) region of Fasciola spp

In naturally infected sheep, all animals were infected and the mean epg was $84.4 \pm 58.3$ on day 0 of the experiment; however, on $30 \mathrm{dpt}$ all sheep had a value of 0 epg [8]. Using the LAMP assay the infection was confirmed in all animals before the treatment although at $30 \mathrm{dpt}$ two sheep resulted positive (Fig. 5a). With the standard PCR, we also found all positive sheep on day 0 although after treatment we only confirmed the infection in one out of the two sheep diagnosed by the LAMP assay (Fig. 5b).

\section{Discussion}

Our aim was to develop a qualitative LAMP assay for the detection of $F$. hepatica in faeces, with the aim to improve certain properties associated with PCR, such as being a time-consuming method that requires complex devices. In the current study, the standard PCR took around $3 \mathrm{~h}$ to obtain a result, comparing with $1 \mathrm{~h}$ and $10 \mathrm{~min}$ with the LAMP assay. On the other hand, the advantage of this qualitative method is that the reaction can be carried out on a block heater and it allows an easy visualisation of the result using fluorescence. Quantitative LAMP assays have also been described although expensive devices are needed. In these assays the reaction was translated on to a real-time PCR platform [9]

Table 2 LAMP primer sequences. F3, forward outer primer; B3, reverse outer primer; FIP, forward inner primer (comprising F1C and F2 sequences); BIP, reverse inner primer (comprising B1C and B2 sequences)

\begin{tabular}{ll}
\hline Primers & Sequence $\left(5^{\prime}\right.$-3') \\
\hline F3 & GCTGGCGTGATCTCCTCTA \\
B3 & AACGTGCCTGGTATGGAATT \\
FIP (F1C-F2) & TCTGCCAAGACAAGGGTGCAT-GTGAGGTGCCAGATCTATGG \\
BIP (B1C-B2) & GTGCAGTGGCGGAATCGTGG-TGTGCCGACTAGGGGATC \\
\hline
\end{tabular}

or results were monitored measuring the turbidity through spectrophotometric analysis [10].

This LAMP assay was designed to detect the infection by any species of Fasciola in faeces, although we only have tested it in sheep infected with $F$. hepatica.

The sensitivity of the LAMP assay and the standard PCR was $1 \times 10^{-3} \mathrm{ng}$ or $10 \mathrm{pg}$. Similar results were described by $\mathrm{Li}$ et al. [10], who reported a limit of detection of $0.3 \times 10^{-3} \mathrm{ng}$ for a LAMP assay to detect Trichinella spiralis and $3.6 \times 10^{-3} \mathrm{ng}$ by means of a standard PCR using also the outer primers. However, Melville et al. [9] showed that the sensitivity to detect the infection by $H$. contortus in faecal samples from sheep was $10^{-5} \mathrm{ng} / \mu \mathrm{l}$. Ai et al. [11] carried out a species specific LAMP assay to discriminate between $F$. hepatica and F. gigantica showing a limit of detection of $10^{-5}$ ng;
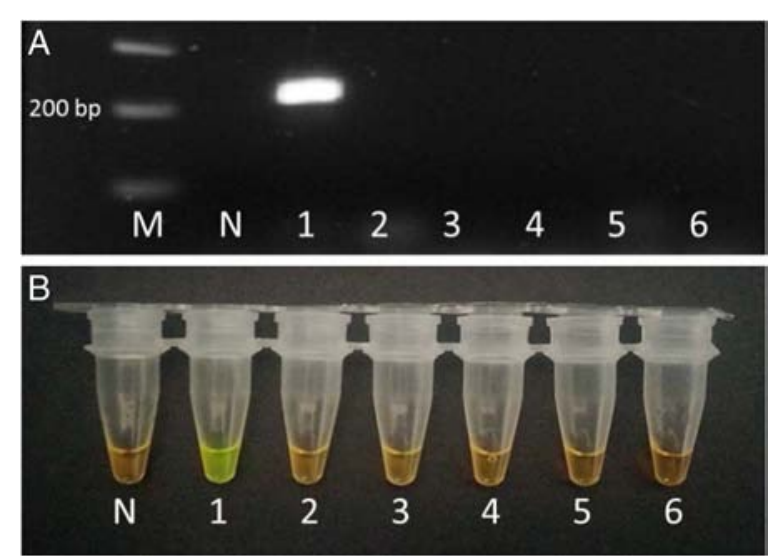

Fig. 2 Specificity of standard PCR with the primers $F 3$ and B3 of the LAMP assay (a) and LAMP assay (b). M: marker. N: negative control. DNA samples from Fasciola hepatica (1), Dicrocoelium dendriticum (2), Calicophoron daubneyi (3), Teladorsagia circumcincta (4), Haemonchus contortus (5) and Trichostrongylus colubriformis (6) 


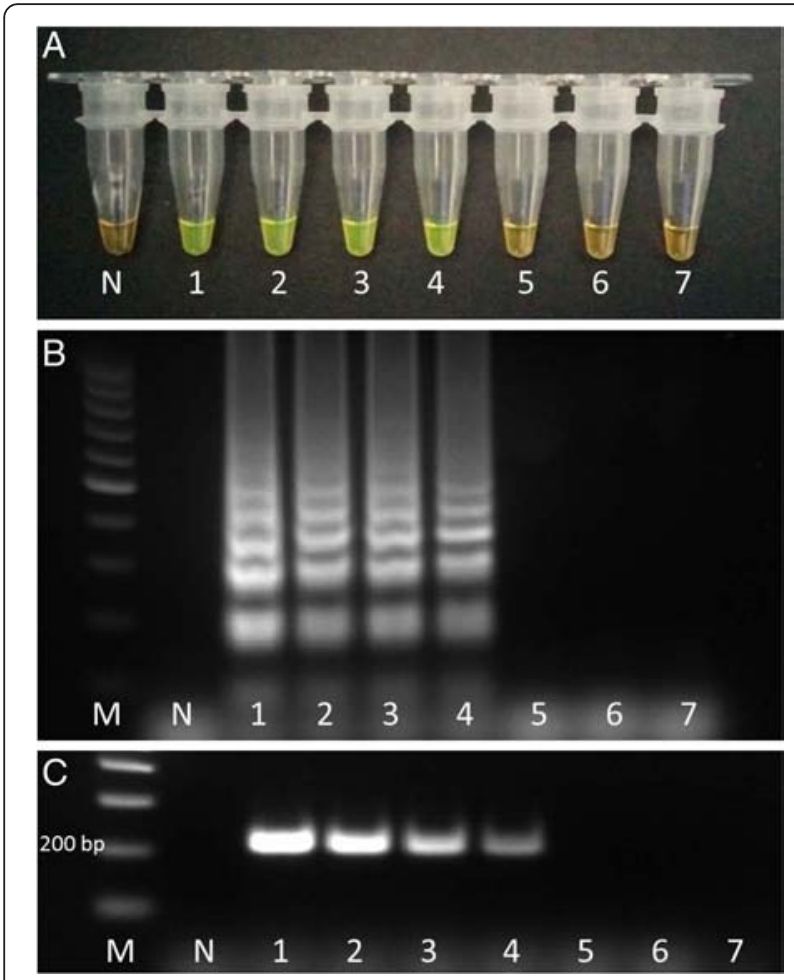

Fig. 3 LAMP assay, 10-fold serial dilution of DNA from F. hepatica. a LAMP reaction after adding SYBR Green I; $\mathbf{b}$ LAMP reaction products analysed by electrophoresis in $2 \%$ agarose gel; c Standard PCR with the primers F3 and B3 of the LAMP assay. N: negative control; 1-7: 10-fold serial dilution of DNA from $F$. hepatica adult worm ranging from $1 \mathrm{ng} / \mathrm{ml}$ (1) to $10^{-6} \mathrm{ng} / \mathrm{ml}$ (7); M: marker

these primers could be tested in faecal samples to distinguish between species in epidemiological studies.

In experimentally infected sheep, we were able to diagnose the infection from the first week pi onward with both techniques, the LAMP assay and the standard PCR. The DNA detected at this stage of the infection could proceed from cellular material from

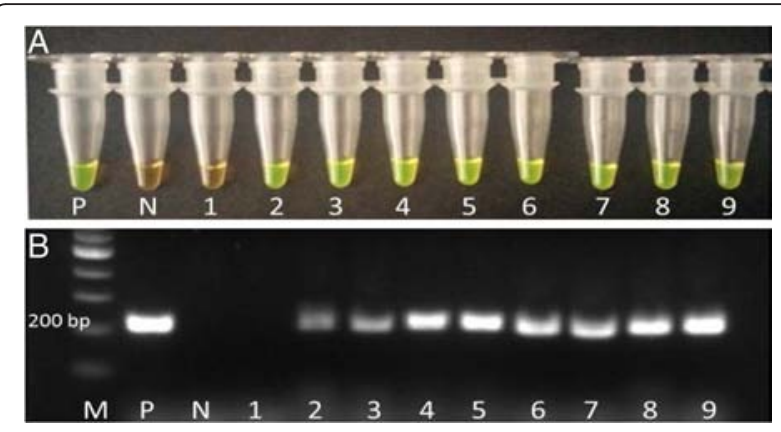

Fig. 4 Detection of the infection in experimentally infected sheep. a LAMP reaction after adding SYBR Green I; $\mathbf{b}$ Standard PCR with the primers F3 and B3 of the LAMP assay. P: positive control; N: negative control; 1-9: pooled samples of sheep on day 0 of the infection (1) and between 1 and 8 weeks post infection (2-9); M: marker

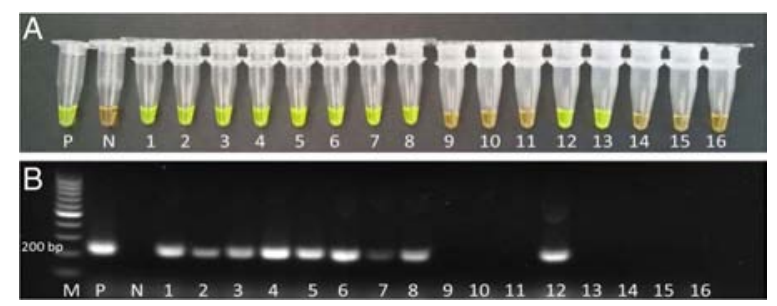

Fig. 5 Detection of the infection in naturally infected sheep. a LAMP reaction after adding SYBR Green I; $\mathbf{b}$ Standard PCR with the primers F3 and B3 of the LAMP assay. P: positive control; N: negative control; 1-8: individual samples of infected sheep on day 0 of treatment; 9-16: individual samples of infected sheep on day 30 post-treatment; M: marker

tegumental turnover and repair during host's immune response [12] or maybe from metacercariae that did not progress. Previously, the detection of $F$. hepatica was possible from two wpi using a nested and another standard PCR with DNA from faeces $[8,12]$.

We tested the LAMP assay with DNA samples of naturally infected sheep previously analysed by RoblesPérez et al. [8]. We found that all animals were infected by $F$. hepatica using the LAMP assay and the standard PCR, in accordance with the result obtained by these authors, who used a different pair of primers to amplify a region of the ITS2. In samples collected at $30 \mathrm{dpt}$, we detected the infection in two out of eight sheep using the LAMP assay, although only in one of these two sheep using the standard PCR. However, Robles-Pérez et al. [8] did not find any positive sheep after treatment using PCR, sandwich ELISA, or direct visualisation of eggs in faeces. Occasionally, fluke eggs can be detected in faeces as false positives due to their presence in the gall-bladder some days after successful treatment [13]. Accordingly, the molecular techniques could amplify DNA from these residual eggs. The positive animals at $30 \mathrm{dpt}$ suggest that the sensitivity of the LAMP assay is slightly higher than the standard PCR; however, these results do not confirm the resistance to triclabendazole since the slaughter of sheep to determine its efficacy was not possible.

\section{Competing interests}

The authors declare that they have no competing interests.

\section{Authors' contributions}

MMV assisted with experimental design, conducted the experiments, and drafted the initial manuscript. FARV assisted with experimental design, offered general expertise and advice and produced the final version of the manuscript. Both authors read and approved the final version of the manuscript.

\section{Acknowledgements}

We thank Drs Manga-González and González-Lanza (Instituto de Ganadería de Montaña, CSIC-ULE, Spain) for providing DNA samples of D. dendriticum and C. daubneyi. This study has been funded by the national project INIA (Instituto Nacional de Investigaciones Agrarias: RTA2010-00094-C03-02), 
Ministry of Economy and Competitiveness (Ministerio de Economia y Competitividad), and also co-funded by the European Regional Development Fund (Fondos Feder).

Received: 11 October 2015 Accepted: 31 January 2016

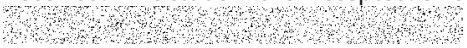

\section{References}

1. Rojo-Vázquez FA, Meana A, Valcárcel F, Martínez-Valladares M. Update on trematode infections in sheep. Vet Parasitol. 2012;30:15-38.

2. Martínez-Valladares M, Robles-Pérez D, Martínez-Pérez JM, CorderoPérez C, Famularo MR, Fernández-Pato N, et al. Increase in prevalence of gastrointestinal nematodes and Fasciola hepatica infections in sheep in the Northwest of Spain: relation to climatic conditions and/or man-made environmental modifications? Parasit Vectors. 2013;27:282-90.

3. Boray JC. Trematode infections of domestic animals. In: Boray JC, Martin PJ, Roush RT, editors. Chemotherapy of Parasitic Diseases. New York: Plenum Press; 1986. p. 401-25.

4. Anderson N, Luong TT, Vo NG, Bui KL, Smooker PM, Spithill TW. The sensitivity and specificity of two methods for detecting Fasciola infections in cattle. Vet Parasitol. 1999;83:15-24.

5. Martínez-Valladares M, Cordero-Pérez C, Castañón-Ordóñez L, Famularo MR, Fernández-Pato N, Rojo-Vázquez FA. Efficacy of a moxidectin/triclabendazole oral formulation against mixed infections of Fasciola hepatica and gastrointestinal nematodes in sheep. Vet Parasitol. 2010;174:166-9.

6. Martínez-Pérez JM, Robles-Pérez D, Valcárcel-Sancho F, González-Guirado AM, de Castro IC, Nieto-Martínez JM, et al. Effect of lipopolysaccharide (LPS) from Ochrobactrum intermedium on sheep experimentally infected with Fasciola hepatica. Parasitol Res. 2013;112:2913-23.

7. Notomi T, Okayama H, Masubuchi H. Loop-mediated isothermal amplification of DNA. Nucleic Acids Res. 2000;28, E63.

8. Robles-Pérez D, Martínez-Pérez JM, Rojo-Vázquez FA, Martínez-Valladares M. The diagnosis of fasciolosis in faeces of sheep by means of a PCR and its application in the detection of anthelmintic resistance in sheep flocks naturally infected. Vet Parasitol. 2013;197:277-82.

9. Melville L, Kenyon F, Javed S, McElarney I, Demeler J, Skuce P. Development of a loop-mediated isothermal amplification (LAMP) assay for the sensitive detection of Haemonchus contortus eggs in ovine faecal samples. Vet Parasitol. 2014:206:308-12.

10. Li X, Liu W, Wang J, Zou D, Wang X, Yang Z, et al. Rapid detection of Trichinella spiralis larvae in muscles by loop-mediated isothermal amplification. Int J Parasitol. 2012;42:1119-26.

11. Ai L, Li C, Elsheikha HM, Hong SJ, Chen JX, Chen SH, et al. Rapid identification and differentiation of Fasciola hepatica and Fasciola gigantica by a loop-mediated isothermal amplification (LAMP) assay. Vet Parasitol. 2010;174:228-33.

12. Martínez-Pérez JM, Robles-Pérez D, Rojo-Vázquez FA, Martínez-Valladares M. Comparison of three different techniques to diagnose Fasciola hepatica infection in experimentally and naturally infected sheep. Vet Parasitol. 2012;190:80-6

13. Flanagan A, Edgar HWJ, Gordon A, Hanna REB, Brenan GP, Fairweather I. Comparison of two assays, a faecal egg count reduction test (FECRT) and a coproantigen reduction test (CRT), for the diagnosis of resistance to triclabendazole in Fasciola hepatica in sheep. Vet Parasitol. 2011;176:170-6.

\section{Submit your next manuscript to BioMed Central and we will help you at every step:}

- We accept pre-submission inquiries

- Our selector tool helps you to find the most relevant journal

- We provide round the clock customer support

- Convenient online submission

- Thorough peer review

- Inclusion in PubMed and all major indexing services

- Maximum visibility for your research

Submit your manuscript at www.biomedcentral.com/submit
Biomed Central 